\title{
Komplexe Fußdeformitäten des Rheumatikers - wie operieren?
}

\author{
Moderne Basismedikamente haben zwar die Häufigkeit schmerzhafter Veränderungen der Füße \\ von Patienten mit rheumatoider Arthritis stark reduziert. Dennoch können im Verlauf auftretende \\ Deformitäten des Vorfußes eine operative Versorgung erfordern.
}

\begin{abstract}
Ursache der Deformitäten vor allem des Vorfußes ist die Synovialitis, welche unter anderem eine intraartikuläre Gelenkkapseldistension und letztlich Gelenkinstabilitäten begünstigt, wie Dr. Manfred Kemmerling, Klinik für Orthopädie, Rheumatologie und Traumatologie, Helios Klinik Attendorn, im Rahmen der Sitzung „Die komplexe Fußdeformität des Rheumatikers, ein Update" erklärte.
\end{abstract}

》) Sind nur einzelne Mittelfußköpfchen betroffen, können gelenkerhaltende Verfahren zum Einsatz kommen

Lassen sich die knöchernen Deformitäten mit Verlust des Längs- und Quergewölbes, Spreizfuß, Hallux valgus, Krallenzehen, Kleinzehendeformitäten und Luxation des Zehengrundgelenke sowie die begleitenden Weichteilveränderungen mit Verlust des plantaren Fettgewebes, mit plantaren Druckschwielen, Synovialfistelungen und zum Teil Perforation nicht verhindern beziehungsweise sind sie nicht konservativ schuhtechnisch versorgbar, besteht bei drohender Immobilität laut Kemmerling die Indikation für eine Operation in Form einer Resektionsarthroplastik.

\section{Heute oft Resektionsarthroplastik MTP II-V plus Lapidusarthrodese} Wegen der bei rheumatischer Vorfußdeformität oft vorliegenden Plantarisierung der Metatarselköpfchen war die Resektionsarthroplastik nach Hoff-
mann/Tillmann (MTP II-V) und Hueter/ Mayo (MTP I) lange Zeit die Operationsmethode der Wahl. Ihr Nachteil war einerseits eine Instabilität des ersten Strahls mit resultierenden Hallux valgus-Rezidiven. Andererseits führte sie aufgrund der Köpfchenresektion nicht selten zu einer "funktionellen Amputation", so Kemmerling.

Daher werde heute neben der Resektionsarthroplastik der MTP IIV-Köpfchen zur Stabilisierung des Vorfußes die Großzehengrundgelenksarthrodese und bei zusätzlicher Instabilität des Pes plantovalgus auch eine Korrekturarthrodese des TMT 1-Gelenkes, die so genannte Lapidusarthrodese, durchgeführt. So kann eine anatomisch korrekte Ausrichtung des ersten Strahls mit gutem Bodenkontakt und damit, die für den Abrollvorgang wichtige Belastbarkeit gewährleistet werden, berichtete der Orthopäde.

\section{Gelenkerhaltende rekonstruktive Verfahren}

Wenn dagegen bei Patienten mit gut kontrollierter Krankheitsaktivität nur einzelne Mittelfußköpfchen betroffen sind und nur eine geringe knöcherne Destruktion vorliegt, können gelenkerhaltende rekonstruktive Verfahren zum Einsatz kommen, beispielsweise die achskorrigierende Scarf- und ChevronOsteotomie am ersten Strahl oder eine verkürzende Weichteil-Osteotomie am zweiten bis fünften Strahl mit Streck- sehnenverlängerung und Synovektomie.

\) Ein Ziel der Operation ist die Wiederherstellung eines harmonischen Alignements aller Metatarsalia

Ziele der Operation sind in jedem Fall ein präzises Weichteilbalancing und die Wiederherstellung eines harmonischen Alignements aller Metatarsalia. Dies soll dem Patienten ein schmerzfreies Abrollen über den Vorfuß mit aktivem Bodenkontakt der Zehen und das Tragen von Konfektionsschuhen mit Einlagen ermöglichen.

rheuma plus $2019 \cdot 18: 205$

https://doi.org/10.1007/s12688-01900303-8

(c) Springer-Verlag GmbH Austria, ein Teil von Springer Nature 2019

Quelle: SpringerMedizin.de, Dr. Wiebke Kathmann, basierend auf dem 47. Kongress der Deutschen Gesellschaft für Rheumatologie (DGRh), 4.-7. September 2019, Dresden

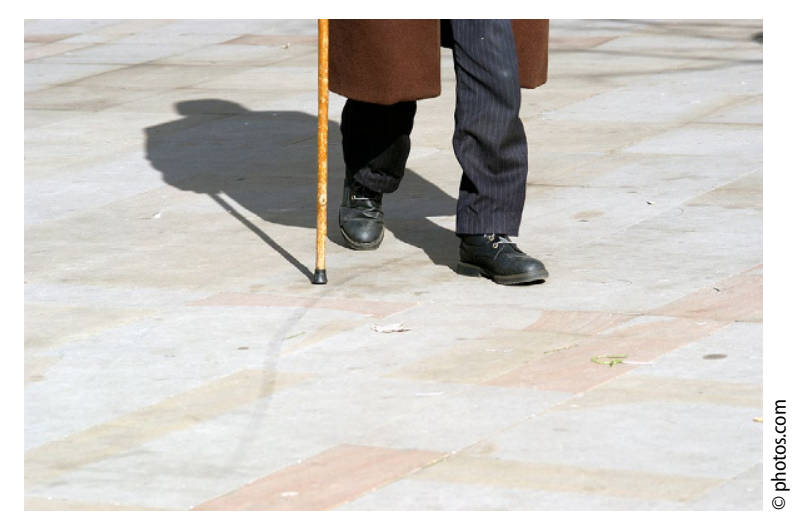

Abb. $1 \wedge$ Orthopädische Eingriffe berücksichtigen inzwischen auch eine anatomisch korrekte Ausrichtung des ersten Strahls mit gutem Bodenkontakt und der für den Abrollvorgang wichtigen Belastbarkeit (Symbolbild mit Fotomodell) 